\title{
Determinants of surgical morbidity in gastric cancer: experience of a single center and literature review
}

\author{
Houyem Mansouri ${ }^{1}$, Ines Zemni ${ }^{2,3}$, Mohamed Ali Ayadi ${ }^{2,3}$, Ines Ben Safta ${ }^{2,3}$, Tarek Ben Dhiab ${ }^{2}$, Najet \\ Mahjoub ${ }^{4}$, Leyla Achouri ${ }^{1}$, Khaled Rahal ${ }^{2}$
}

\begin{abstract}
${ }^{1}$ Department of Surgical Oncology, Regional hospital of Jendouba, University Tunis El Manar, Faculty of Medicine of Tunis, Tunisia.
${ }^{2}$ Department of Surgical Oncology, Salah Azaiez Institute, University Tunis El Manar, Faculty of Medicine of Tunis, Tunisia.

${ }^{3}$ Laboratory of Microorganisms and active Bio-molecules (LR03ES03), Faculty of Sciences of Tunis- University of Tunis El Manar, Tunis, Tunisia.

${ }^{4}$ Department of Oncology, Regional hospital of Jendouba, University Tunis El Manar, Faculty of Medicine of Tunis, Tunisia.
\end{abstract}

\section{To Cite}

Mansouri H, Zemni I, Ali Ayadi M, Ben Safta I, Ben Dhiab T, Mahjoub N, Achouri L, Rahal K. Determinants of surgical morbidity in gastric cancer: experience of a single center and literature review. J Gastric Surg 2020; 2(4).

\section{Publication history}

Received: December 5, 2020

Accepted: December 12, 2020

Article in press: December 13, 2020

Published online: December 15, 2020

\section{*Correspondence to}

Dr. Ines Zemni

Department of surgical oncology

Salah Azaiez Institute

University Tunis El Manar

Faculty of Medicine of Tunis

Avenue 9 April Bab Saadoun

1006 Tunis, Tunisia

ines.zemni@yahoo.fr

Telephone: +216 25560736

\section{ABSTRACT \\ Background:}

This study aimed to evaluate the severity of intraoperative and post-operative complications of gastric cancer surgery and to investigate the predictive factors correlated to surgical morbidity.

Methods:

We included 145 patients operated for gastric cancer. We investigated the risk factors associated with complications, length of hospital stay, operative time, and intraoperative blood transfusion (BT). Significant risk factors were analyzed by multiple logistic regression analysis.

Results:

Postoperative complications occurred in 32 patients $(22.1 \%)$ and the rate of major complications was $7.6 \%$. The rate of anastomotic fistula was $6.9 \%$ and was correlated to diabetes, tumor size, operative time, surgical margin, and extended lymphadenectomy. The mean risk factors for postoperative morbidity were the presence of comorbidities and ASA score $(\mathrm{p}=0.021)$, intraoperative BT $(\mathrm{p}=0.045)$ and prolonged operative time $(\mathrm{p}=0.055)$.

Conclusion:

Surgical morbidity of gastric cancer is correlated to the extent of resection as well as the clinical and histological characteristics.

Key Words:

gastric cancer, surgery, Morbidity, postoperative complications, Prognosis. 


\section{Background}

Management of gastric cancer has undergone in the last decade remarkable progress both in the neo-adjuvant and adjuvant treatments and in the surgical procedure as regards the extent of resection and the lymph node dissection. These advances are certainly associated with an improvement in the overall prognosis of the neoplastic disease. But this gain has been associated with over- morbidity and mortality that we need to know to prevent it. The rate of postoperative morbidity reported by the largest randomized controlled series varies from 11 to $46 \%$.[1] This significant variability in the literature is explained not only by the differences in the characteristics of the studied populations, the therapeutic procedures but also by the definition of the morbidity used in each study. Thus, to limit the subjectivity in the analysis of the complications, many authors used the Clavien and Dindo classification of postoperative complications published in 2004. This classification seems more straightforward, objective, but above all reproducible, since it evaluates the severity of the complications according to their therapeutic management.[2] This study aimed to assess the severity of intraoperative and postoperative complications of gastric surgery and to investigate the predictive factors correlated to surgical morbidity.

\section{Methods}

Approval to conduct this study was obtained from the Institutional Review Board of the Salah Azaiz Institute. We conducted a retrospective study including 145 patients with histologically proven gastric adenocarcinoma who underwent curative or palliative gastrectomy from January 2005 to December 2015. Noninclusion criteria were as follows:(1) metastatic disease, (2) other synchronous tumoral location, (3) unknown surgery status, (4) unknown vital status; (5) incomplete pathological data.

After institutional review board approval, we collected patient and tumor-related factors through medical records: age, sex, BMI, proteinemia (hypoproteinemia was defined as less than $60 \mathrm{~g} / \mathrm{dl}$ ), the hemoglobin level, comorbidities, and American Society of Anesthesiologist (ASA) score, tumor size, depth of invasion and the stage of gastric cancer according to the eighth edition of the American Joint Committee on Cancer TNM classification system.

The surgical variables included the type of procedure (total versus partial gastrectomy), the extent of lymph node dissection (D0, D1.5, and D2) based on the Japanese Gastric Cancer Treatment Guidelines 2010 (version 3)[3] and the number of retrieved lymph nodes (NRLN), multivisceral organ resection (MVR), operative time measured from the first skin incision to the closure of all skin incisions and confirmed by the attending anesthesiologist in the operating room, intraoperative allogeneic blood transfusion and the length of hospital stay.

The extent of the lymphadenectomy was based on the individual surgeon's judgment. Splenectomy was performed in cases of metastatic lymph nodes at the hilum of the spleen or because of iatrogenic injury. The extent of stomach resection was related to the primary tumor site: total gastrectomy was performed in all proximal tumor locations and total gastric tumors, and subtotal gastrectomy was performed for distal tumor locations, provided that a 5 to $6-\mathrm{cm}$ safety margin was present. A multi-visceral resection was performed in all cases of T4 or suspected T4 tumors. After total gastrectomy, reconstruction was made by Roux-en-Y esophagojejunostomy. Esophago-jejunal anastomoses were performed by hand-suture or mechanically with a circular stapler according to the surgeon's choice. The stapled esophageal and jejunal doughnuts were examined for completeness of anastomosis. A hand-sutured or stapler Billroth II followed subtotal gastrectomy. A prophylactic antibiotic of a second- or third-generation cephalosporin was administered to all patients and usually lasted for 5 days following the operation.

The integrity of the anastomosis was routinely evaluated on the seventh postoperative day by a water-soluble contrast swallow and/or by ingestion of methylene blue before reintroducing an oral intake. The anastomotic fistula was diagnosed with extravasation of contrast product during radiological examination and/or with discharge or gastrointestinal content through a drain. Clinical leakage was defined as the presence of either clinical symptoms suggesting potential leakages such as abdominal pain abnormal with drain discharge, fever, and leukocytosis.

Postoperative morbidity and mortality included all adverse events reported within the first 30 days after surgery or during the same hospitalization, and late complications were not included in this study. Surgical complications included anastomotic fistula and leakage, early anastomotic stenosis, septic collection, and wound infection, pleural effusion, ileus, and bleeding. Non-surgical complications were heart failure, pneumonia or respiratory failure, urinary tract infection, decompensation of diabetes, and thromboembolic events. All complication data were graded retrospectively according to the Clavien-Dindo classification from Grade 1 to Grade 5 according to the treatment for each complication. Major complications included all complications classified as grade III A or more according to the Clavien Dindo system.

\section{Statistical analysis}

The categorical variables were presented as numbers and percentages. Continuous variables were expressed as the mean \pm standard Deviation (SD) with ranges, and groups were compared using the Test $t$ of Student. Moreover, some continuous variables were converted to dichotomous variables for convenience, including the age ( $\leq 65$ years versus $>65$ years), the body mass index BMI $(<20 \mathrm{~kg} / \mathrm{m} 2$ versus $\geq 20 \mathrm{~kg} / \mathrm{m} 2)$, hemoglobin rate $(\leq 10 \mathrm{~g} / \mathrm{dl}$ versus $>10 \mathrm{~g} / \mathrm{dl})$, the tumor size $(<50 \mathrm{~mm}$ versus $\geq 50 \mathrm{~mm})$ and the NRLN $(\leq 15,16-25$ and $\geq 25)$. Clinical and pathological data were compared between patients to analyze the major risk factors associated with complications, length of hospital stay, operative time and intraoperative blood transfusion. The groups were compared using the chi-square test or Fisher's exact test. All variables with a p-value $<0.2$ in the univariate analysis were entered into a multivariate analysis using 
a logistic regression model to identify independent factors of perioperative blood transfusion and operative time. We used the Statistical Package for Social Science (SPSS), version 20.0 for Windows, and ap-value less than 0.05 was considered significant.

\section{Results}

The clinicopathological characteristics of the patients are summarized in Table 1. There were 93 males $(64 \%)$ and 52 females ( $36 \%$ ), with a mean age of 61.48 years \pm 12.86 (range, 26 to 85 years) and 44 patients $(49.5 \%$ ) were more than 70 years old.

The operative data are summarized in Table 2. Total gastrectomy was performed in 77 patients $(53.1 \%)$ and subtotal gastrectomy in 68 patients $(46.9 \%)$. Combined organ resection was performed in $34(23.4 \%)$ patients. D1 lymph node dissection was carried out in 15 cases, and extended lymphadenectomy in 130 patients $(89.7 \%)$ from which 36 patients $(24.8 \%)$ had D1.5 lymphadenectomy and 94 patients had D2 lymphadenectomy.

The Mean operative time was 194 minutes \pm 53.22 with extremes ranging from 110 to $350 \mathrm{mn}$. Univariate analysis of the factors influencing the operating time is shown in Table 3. The mean operative time was significantly increased in patients with proximal and middle third tumors compared with distal tumors ( $208.81 \mathrm{~mm}$ vs $181.42 \mathrm{mn}$ respectively, $\mathrm{p}=0.002)$. Tumor staged T3-T4 required a longer operative time compared to pT1-T2 tumors (199.81mnvs $178.85 \mathrm{mn}$ respectively, $\mathrm{p}=0.032$ ). We also found that the mean operative time was significantly longer with total gastrectomy compared to partial gastrectomy (204.16mnvs $182.56 \mathrm{mn}$ respectively, $\mathrm{p}=0.015)$, multi-organ resection $(215 \mathrm{mn}$ vs $187.60 \mathrm{mn}, \mathrm{p}=0.021)$, D2 dissection compared to D1 / D1.5 dissection $(204.02 \mathrm{~mm}$ vs $188.61 \mathrm{mn}$ respectively, $\mathrm{p}=0.004)$ and in case of splenectomy or splenopancreatectomy $(\mathrm{p}=0.037)$. On multivariate analysis extended lymphadenectomy and combined organ resection were the only independent factors associated with a longer operative time.

Intraoperative blood transfusion was performed in 77 patients $(53.1 \%)$, with a mean amount of 2.14 units and extremes ranging from 1 to 4 units. Of the transfused patients, 19 patients $(24.7 \%)$ required more than three units. Univariate analysis of factors associated with intraoperative blood transfusion is shown in table4. Transfused patients had a longer mean operative time compared to non-transfused patients $(23.04 \mathrm{mn}$ vs $183.83 \mathrm{mn}, \mathrm{p}=0.054)$. Significant factors on the univariate analysis were included in the multivariate analysis (Table4). Tumor location and combined organ resection were found to be the independent risk factors of intraoperative blood transfusion.

A total of 113 out of $145(77.9 \%)$ patients had no complications, and $32(22.1 \%)$ had at least one medical or surgical complications. Details of postoperative complications are listed in Table 2. Eighteen patients $(12.4 \%)$ had one or more medical complications of which seven patients had significant medical complications. They were dominated by postoperative pneumonia occurring in 13 cases $(9 \%)$ and pulmonary embolism in 2 cases $(1.4 \%)$.

Surgical complications occurred in 18 patients within a delay of 10 days (range, 4 to 20 days). The anastomotic fistula was the most common surgical complication occurring in 10 cases $(6.9 \%)$, and postoperative peritonitis secondary to complete anastomotic leakage was diagnosed in only one patient. From the 145 patients, four $(2.8 \%)$ required re-laparotomies for serious complications: one case of stenosis of the esophagojejunal anastomosis, one case of anastomotic leakage, one case of the esophago-jejunal fistula with major ionic disorders and severe dehydration, and one case of a deep abdominal abscess that was not accessible to percutaneous radiologic drainage.

Using the Clavien-Dindo classification, the 32 complicated patients had the following grades: 21 patients $(14.5 \%)$ had grade II, 3 patients $(2.1 \%)$ had grade IIIa, 2 patients $(1.4 \%)$ had grade IIIB and one $(1.6 \%)$ had grade IVa. Postoperative death (grade V of Clavien-Dindo classification) occurred in five patients (3.4\%) within a mean delay of 30 days after surgery (range 18 to 74 days). Three patients died because of anastomotic fistula, one patient died after reoperation for anastomotic stenosis and the last patient died from respiratory failure secondary to pneumonia.

Univariate analysis of clinical and therapeutic predictive factors of postoperative complications is shown in Table 5 . We found that the mean risk factors for postoperative morbidity were the presence of comorbidities $(\mathrm{p}=$ $0.021)$, intraoperative blood transfusions $(p=0.045)$ and prolonged operative time $(\mathrm{p}=0.055)$. Hypoproteinemia at diagnosis seems to increase morbidity ( $28.8 \%$ vs $18.3 \%$, $p=0.066$ ). Patients with BMI greater than or equal to 20 had a higher rate of complications than those with a BMI of less than $20(23.3 \%$ vs. $20.8 \%, p=0.842)$. However, we found that age, gender, locally advanced tumors, extended lymphadenectomy, splenectomy or SPC, total gastrectomy, and combined organ resection were not significantly associated with higher postoperative morbidity.

The mean length of postoperative hospital stay was 15.08 days \pm 7.48 (range 7-74 days). Univariate analysis of factors associated with the length of hospital stay is represented in Table 6. No significant difference was found in the period of hospital stay according to the extent of gastrectomy and lymphadenectomy. However, the duration of hospitalization was significantly increased in patients with comorbidities regardless of age and gender $(p=0.017)$. Patients who presented postoperative complications had a more extended hospital stay $(\mathrm{p}<0.001)$. According to Clavien-Dindo classification, the length of hospital stays progressively increased from non-complicated patients to grade $\mathrm{V}$ patients $(\mathrm{P}<0.0001)$. The duration of hospitalization is extended by 13.99 days $(p=0.03)$ in case of anastomotic fistula and by 6.17 days $(p=0.004)$ in cases of respiratory infections.

The results of the univariate analysis of patient-related risk factors of fistula and leakage showed that these complications were more frequent in diabetic patients $(23.5 \%$ vs $4.7 \% ; \mathrm{p}=0.004)$. According to laboratory variables, preoperative hypoproteinemia $(11.5 \%$ in case of hypoproteinemia vs $4.3 \%$ with normal proteinemia, $\mathrm{p}=0.168$ ) and anemia with hemoglobin rate less than $10 \mathrm{~g} / \mathrm{dl}(12.5 \%$ vs $4.8 \%, \mathrm{p}=0.139)$ were likely to be associated 
with a higher rate of fistula even if the difference was not statistically significant. In the analysis of tumor-related factors, anastomotic fistula was significantly associated with tumor size exceeding $50 \mathrm{~mm}(10.3 \%$ vs $1.7 \%$, $\mathrm{p}=0.045)$, gastric walls rigidity $(33.8 \%$ vs $5.1 \%$; $\mathrm{p}=0.016)$, and the length of the proximal margin of resection (the mean length of the proximal margin in patients with fistula was $41 \pm 24.358 \mathrm{~mm}$ vs $57.55 \pm 26.008, \mathrm{p}=0.042$ ). When analyzing surgical related factors, we found that the type of gastrectomy, the extent of lymphadenectomy, and combined organ resection were not significantly associated with a more frequent fistula. Nevertheless, the number of retrieved lymph nodes $(23.24 \pm 11.036$ vs $27.5 \pm 12.059$ in case of anastomotic fistula, $p=0.073)$, as well as the duration of the operation (190.77 \pm 12.59 min vs $238 \pm 16.96 \mathrm{~min}$ in case of fistula, $p=0.006)$, were identified as predictive factors of fistula.

Table 1: Clinical and histological patients' characteristics

\begin{tabular}{|c|c|c|c|}
\hline Variables & & $\mathbf{N}$ & $\%$ \\
\hline \multirow{3}{*}{ Age (mean, $\pm \mathrm{DS}, \min$, max, ans) } & & \multicolumn{2}{|c|}{$61.48 \pm 12.86(26-85)$} \\
\hline & $\geq 70$ years & 44 & 30.3 \\
\hline & $<70$ years & 101 & 69.7 \\
\hline \multirow[t]{2}{*}{ Gender } & Male & 93 & 64 \\
\hline & Female & 52 & 36 \\
\hline \multirow{3}{*}{ BMI (mean \pm SD,kg/m2) } & & $19[12.48-2$ & \\
\hline & $<20 \mathrm{~kg} / \mathrm{m} 2$ & 72 & 49.7 \\
\hline & $\geq 20 \mathrm{~kg} / \mathrm{m} 2$ & 73 & 50.3 \\
\hline \multirow[t]{2}{*}{ Hypoproteinemia } & No & 93 & 64.1 \\
\hline & Yes & 52 & 35.9 \\
\hline \multirow[t]{2}{*}{$\mathrm{Hb}(g / d l)$} & $\leq 10 \mathrm{~g} / \mathrm{dl}$ & 40 & 27.6 \\
\hline & $>10 \mathrm{~g} / \mathrm{dl}$ & 105 & 72.5 \\
\hline \multirow[t]{2}{*}{ Comorbidities } & No & 101 & 69.7 \\
\hline & Yes & 44 & 30.3 \\
\hline \multirow[t]{2}{*}{ ASA score } & $<$ ASA 3 & 119 & 82. \\
\hline & $\geq$ ASA 3 & 26 & 17.9 \\
\hline \multirow[t]{4}{*}{ Tumor location } & Upper third & 21 & 14.5 \\
\hline & Middle third & 45 & 31 \\
\hline & Distal third & 77 & 53.1 \\
\hline & Pangastric tumor & 2 & 1.4 \\
\hline \multirow{2}{*}{$\begin{array}{l}\text { Tumor size } \\
\text { (mean } \pm \mathrm{DS}, \min , \max , \mathrm{mm})\end{array}$} & & $49[12-220 n$ & \\
\hline & $\begin{array}{l}<50 \mathrm{~mm} \\
\geq 50 \mathrm{~mm}\end{array}$ & $\begin{array}{l}58 \\
87\end{array}$ & $\begin{array}{l}40 \\
60\end{array}$ \\
\hline \multirow[t]{3}{*}{ Lauren Classification } & Intestinal & 109 & 75.2 \\
\hline & Mixed & 4 & 2.8 \\
\hline & Diffuse & 32 & 22.1 \\
\hline \multirow[t]{3}{*}{ Differenciation } & Well & 63 & 43.4 \\
\hline & Meanly & 47 & 32.4 \\
\hline & Poorly/undifferenciated & 35 & 24.2 \\
\hline \multirow[t]{2}{*}{ LVI } & No & 73 & 50.3 \\
\hline & Yes & 72 & 49.7 \\
\hline \multirow[t]{2}{*}{ PNI } & No & 76 & 52.4 \\
\hline & Yes & 69 & 47.6 \\
\hline \multirow[t]{4}{*}{ pT stage } & pT1 & 8 & 5.5 \\
\hline & рT2 & 32 & 22.1 \\
\hline & рT3 & 61 & 42.1 \\
\hline & pT4 & 44 & 30.3 \\
\hline LN status & $\mathrm{N}-$ & 26 & 17.9 \\
\hline & $\mathrm{N}+$ & 119 & 82.1 \\
\hline pN stage & pNO & 26 & 17.9 \\
\hline & $\mathrm{pN} 1$ & 31 & 21.4 \\
\hline & pN2 & 41 & 28.3 \\
\hline & pN3a & 28 & 19.3 \\
\hline & pN3b & 19 & 13.1 \\
\hline NRLN (mean $\pm D S$, min, max) & 23.6 & $6[5-57$ gan & \\
\hline & $<15$ & 27 & 18.6 \\
\hline & $15-24$ & 61 & 42.1 \\
\hline & $\geq 25$ & 57 & 39.3 \\
\hline NMLN (mean $\pm D S$, min, max) & & [1-38 gang & \\
\hline LNR & LNRO & 26 & 17.9 \\
\hline & LNR1 & 25 & 17.2 \\
\hline & LNR2 & 30 & 20.7 \\
\hline & LNR3 & 64 & 44.2 \\
\hline UICC stage & I & 16 & 11 \\
\hline & II & 45 & 31 \\
\hline & III & 77 & 53 \\
\hline & IV & 7 & 5 \\
\hline Resection & Ro & 136 & 93.8 \\
\hline & R1 & 8 & 5.5 \\
\hline & R2 & 1 & 0.7 \\
\hline Treatment sequency & Surgery alone & 47 & 32.4 \\
\hline & CT-Sur -CT/RTCT & 13 & 9 \\
\hline & Sur+CT ADJ & 27 & 18.6 \\
\hline & SUR+ RTCT/RT ADJ & 58 & 40 \\
\hline
\end{tabular}

CT: chemotherapy, ADJ: adjuvant, RTCT/RT: radio-chemotherapy or adjuvant radiation therapy, SUR: surgery, LVI: lympho-vascular invasion, PNI: perineural invasion, NRLN: number of retrieved lymph nodes, NMLN: number of metastatic lymph nodes

LNR : Iymph nodes ratio, SD : standard deviation 
Table 2: Surgical and postoperative features of patients

\begin{tabular}{|c|c|c|}
\hline Variables & $\mathbf{N}$ & Rate \\
\hline \multicolumn{3}{|l|}{ Gastrectomy } \\
\hline Total gastrectomy & 77 & $53.1 \%$ \\
\hline Partial gastrectomy & 68 & $46.9 \%$ \\
\hline Combined organ resection & 34 & $23.4 \%$ \\
\hline Transverse mesocolon resection & 12 & $8.3 \%$ \\
\hline Transverse colectomy & 5 & $3.4 \%$ \\
\hline Small bowel resection & 4 & $2.8 \%$ \\
\hline Liver & 1 & $0.7 \%$ \\
\hline Cholecystectomy & 1 & $0.7 \%$ \\
\hline Cephalic duodenopancreatectomy & 1 & $0.7 \%$ \\
\hline SPC & 3 & $2.1 \%$ \\
\hline Splenectomy & 11 & $7.6 \%$ \\
\hline \multicolumn{3}{|l|}{ Lymphadenectomy } \\
\hline D1 & 15 & $10.3 \%$ \\
\hline D1.5 & 36 & $24.8 \%$ \\
\hline $\mathrm{D} 2$ & 94 & $64.8 \%$ \\
\hline \multicolumn{3}{|l|}{ Number of retrieved lymph nodes } \\
\hline$\leq 15$ & 34 & $23.4 \%$ \\
\hline $16-25$ & 59 & $40.7 \%$ \\
\hline$\geq 25$ & 52 & $35.9 \%$ \\
\hline \multicolumn{3}{|l|}{ Intraoperative blood transfusion } \\
\hline No & 68 & $46.8 \%$ \\
\hline Yes & 77 & $53.1 \%$ \\
\hline Operative time (mean $\pm S D, m n$, range ) & \multicolumn{2}{|c|}{$194 \mathrm{~mm} \pm 53.22[110-350 \mathrm{mn}]$} \\
\hline Length of hospital stay (mean $\pm S D$, days, range ) & \multicolumn{2}{|c|}{$15.08 \pm 7.485[7-74$ days $]$} \\
\hline Postoperative mortality & 5 & $3.4 \%$ \\
\hline Total postoperative complications & 32 & $22.1 \%$ \\
\hline \multicolumn{3}{|l|}{ Surgical complications } \\
\hline Anastomotic fistula & 10 & $6.9 \%$ \\
\hline Isolated duodenal fistula & 1 & $0.7 \%$ \\
\hline Postoperative peritonitis: Anastomotic leakage & 1 & $0.7 \%$ \\
\hline Deep abdominal collection & 3 & $2.1 \%$ \\
\hline Parietal abscess & 3 & $2.1 \%$ \\
\hline Anastomotic stenosis & 2 & $1.4 \%$ \\
\hline Pleural effusion & 2 & $1.4 \%$ \\
\hline \multicolumn{3}{|l|}{ Non surgical complications } \\
\hline Respiratory infection & 13 & $9 \%$ \\
\hline Pulmonary embolism & 2 & $1.4 \%$ \\
\hline Cardiac failure & 1 & $0.7 \%$ \\
\hline Urinary infection & 1 & $0.7 \%$ \\
\hline Diabetic decompensation & 1 & $0.7 \%$ \\
\hline \multicolumn{3}{|l|}{ Clavien-Dindo classification } \\
\hline No complications & 113 & $77.9 \%$ \\
\hline Grade II & 21 & $14.5 \%$ \\
\hline Grade IIla & 3 & $2.1 \%$ \\
\hline Grade IIIb & 2 & $1.4 \%$ \\
\hline Grade Iva & 1 & $0.7 \%$ \\
\hline Grade V & 5 & $3.4 \%$ \\
\hline
\end{tabular}


Table 3: Univariate and multivariate analysis of factors influencing the operative time

\begin{tabular}{|c|c|c|c|c|c|}
\hline \multirow[t]{2}{*}{ Factors } & \multirow[t]{2}{*}{$\mathbf{N}$} & \multicolumn{2}{|c|}{ Univariate analysis } & \multicolumn{2}{|c|}{ Multivariate analysis } \\
\hline & & $\begin{array}{c}\text { Operative time } \\
(\text { Mean } \pm \text { SD, mn) }\end{array}$ & $\mathbf{P}^{*}$ & $\begin{array}{l}\text { Exp B } \\
95 \% \mathrm{Cl}\end{array}$ & $\mathbf{P}$ \\
\hline Tumor location & & & 0.002 & - & - \\
\hline Distal & 77 & $181.42 \pm 52.897$ & & & \\
\hline Others & 68 & $208.31 \pm 50.480$ & & & \\
\hline Tumor size (mm) & & & 0.256 & - & - \\
\hline$\geq 50$ & 87 & $197.15 \pm 53.625$ & & & \\
\hline$<50$ & 58 & $187.84 \pm 52.733$ & & & \\
\hline Depth of invasion & & & 0.034 & -0.196 & 0.160 \\
\hline $\mathrm{T} 1-\mathrm{T} 2$ & 40 & $178.85 \pm 49.207$ & & {$[-5.413-32.511]$} & \\
\hline T3-T4 & 105 & $199.81 \pm 53.918$ & & & \\
\hline Gastrectomy & & & 0.015 & - & - \\
\hline$P G$ & 68 & $182,56 \pm 56,130$ & & & \\
\hline TG & 77 & $204,16 \pm 48,878$ & & & \\
\hline MVR & & & 0.021 & - & - \\
\hline No & 111 & $187,60 \pm 49,186$ & & & \\
\hline Yes & 34 & $215 \pm 61,62$ & & & \\
\hline Splenectomy/ Sp & lenop & ncreatectomy & 0.037 & 0.287 & 0.001 \\
\hline No & 132 & $189.57 \pm 75.622$ & & [22.158 - 80.997] & \\
\hline Yes & 14 & $235.71 \pm 75.622$ & & & \\
\hline Lymphadenecton & & & 0.004 & -0.196 & 0.018 \\
\hline D1/D1.5 & 51 & $188,61 \pm 56,130$ & & {$[-39.918,-3.756]$} & \\
\hline$\geq \mathrm{D} 2$ & 94 & $204,02 \pm 39,586$ & & & \\
\hline
\end{tabular}

Table 4: Univariate and multivariate analysis of clinical, therapeutic and histological factors associated with intraoperative blood transfusion

\begin{tabular}{|c|c|c|c|c|c|c|}
\hline \multirow[t]{2}{*}{ Variables } & \multirow[t]{2}{*}{$\mathbf{N}$} & \multicolumn{2}{|c|}{ Univariate analysis } & \multirow[b]{2}{*}{$\mathbf{p}$} & \multicolumn{2}{|c|}{ Multivariate analysis } \\
\hline & & No BT & BT & & $\begin{array}{c}\text { OR } \\
95 \% \text { IC }\end{array}$ & $\mathbf{P}$ \\
\hline \multicolumn{2}{|l|}{$\begin{array}{l}\text { Age } \\
\text { (mean } \pm S D \text {, years) }\end{array}$} & $60.64 \pm 12.88$ & $62.23 \pm 12.89$ & $0.692 \dagger$ & - & - \\
\hline \multicolumn{2}{|l|}{ Gender } & & & $0.864^{*}$ & - & - \\
\hline Male & 93 & $43(46.2 \%)$ & $50(53.8 \%)$ & & & \\
\hline Female & 52 & $25(48.1 \%)$ & $27(51.9 \%)$ & & & \\
\hline $\begin{array}{l}\text { BMI } \\
\text { (mean } \pm \mathrm{SD}, \mathrm{kg} / \mathrm{m} 2)\end{array}$ & 145 & $20.039 \pm 2.79$ & $19.78 \pm 2.66$ & $0.164 \dagger$ & - & - \\
\hline \multicolumn{2}{|l|}{ Tumor location } & & & $0.008^{*}$ & 0.174 & 0.03 \\
\hline Distal & 77 & $44(57.1 \%)$ & $33(42.9 \%)$ & & {$[0,017-0.330]$} & \\
\hline Others & 68 & 24 (35.3\%) & $44(67.7 \%)$ & & & \\
\hline \multicolumn{2}{|l|}{ Gastrectomy } & & & $0.007^{*}$ & - & NS \\
\hline TG & 77 & $28(36.4 \%)$ & $49(63.6 \%)$ & & & \\
\hline PG & 68 & $40(58.8 \%)$ & $28(41.2 \%)$ & & & \\
\hline MVR & & & & $0.001^{*}$ & 0.267 & 0.001 \\
\hline No & 111 & $61(55 \%)$ & $50(45 \%)$ & & {$[0,131-0.497]$} & \\
\hline Yes & 34 & $7(20.6 \%)$ & $27(79.4 \%)$ & & & \\
\hline \multicolumn{4}{|c|}{ Splenectomy/ Splenopancreatectomy } & $0.002^{*}$ & - & NS \\
\hline No & 131 & $67(51.1 \%)$ & $64(48.9 \%)$ & & & \\
\hline Yes & 14 & $1(7.1 \%)$ & $13(92.9 \%)$ & & & \\
\hline \multicolumn{3}{|l|}{ Lymphadenectomy } & & $0.728^{*}$ & - & - \\
\hline D1 & 15 & $9(60 \%)$ & $6(40 \%)$ & & & \\
\hline D1.5 & 36 & $13(31.6 \%)$ & $23(63.9 \%)$ & & & \\
\hline D2 & 94 & 46 (48.9\%) & $48(51.1 \%)$ & & & \\
\hline $\begin{array}{l}\text { Operative time } \\
\text { (mean } \pm \mathrm{SD}, \mathrm{mn} \text { ) }\end{array}$ & 145 & 183.82 & 203.04 & $0.054 \dagger$ & - & NS \\
\hline \multicolumn{4}{|l|}{ NRLN } & $0.042^{*}$ & 0,132 & 0,097 \\
\hline$\leq 15$ & 34 & $21(61.8 \%)$ & $13(38.2 \%)$ & & {$[-0,016-0.189]$} & \\
\hline $16-25$ & 59 & $27(48.8 \%)$ & $32(54.2 \%)$ & & & \\
\hline$\geq 25$ & 52 & $20(38.5 \%)$ & $32(61.5 \%)$ & & & \\
\hline \multicolumn{4}{|l|}{ Depth of invasion } & $0.116^{*}$ & - & - \\
\hline $\mathrm{T} 1 / \mathrm{T} 2$ & 40 & $23(57.6 \%)$ & $17(42.5 \%)$ & & & \\
\hline T3/T4 & 105 & $45(42.9 \%)$ & $60(57.1 \%)$ & & & \\
\hline $\begin{array}{l}\text { Tumor size } \\
\text { (mean } \pm S D, m m)\end{array}$ & 145 & $51.16 \pm 27.62$ & $71.66 \pm 38.48$ & $0.027 \dagger$ & - & NS \\
\hline \multicolumn{7}{|c|}{$\begin{array}{l}\text { BMI: Body mass index, TG: total gastrectomy, PG : partial gastrectomy, MVR: multivisceral } \\
\text { resection, NRLN: number of retrieved lymph nodes. BT: blood transfusion } \\
\text { † test T of Student. } \\
\text { * test chi2 Pearson } \\
\text { SD: standard deviation }\end{array}$} \\
\hline
\end{tabular}


Table 5: Univariate analysis of predictive factors of postoperative complications

\begin{tabular}{|c|c|c|c|c|c|}
\hline \multirow[t]{2}{*}{ Variables } & & \multirow[t]{2}{*}{$\mathbf{N}$} & \multicolumn{2}{|c|}{ Postoperative complications } & \multirow{2}{*}{$\begin{array}{c}P \\
\text { Value }\end{array}$} \\
\hline & & & Yes & No & \\
\hline Age (mean, years) & & 145 & $61,16 \pm 12,67$ & $62,66 \pm 13,70$ & $0.563 \dagger$ \\
\hline \multirow[t]{2}{*}{ BMI (kg/m2) } & $<20$ & 72 & $57(79.2 \%)$ & $15(20.8 \%)$ & $0.842^{*}$ \\
\hline & $\geq 20$ & 73 & $56(76.3 \%)$ & $17(23.3 \%)$ & \\
\hline Proteinemia (mean, g/l) & & 145 & $61,72 \pm 7,83$ & $58,88 \pm 7,093$ & $0.066^{*}$ \\
\hline \multirow[t]{2}{*}{ Hypoproteinemia } & No & 93 & $76(81.7 \%)$ & $17(18.3 \%)$ & \\
\hline & Yes & 52 & $37(71.2 \%)$ & $15(28.8 \%)$ & \\
\hline Hemoglobin level (g/dl) & & 145 & $10,74 \pm 1,75$ & $10,62 \pm 2,09$ & $0.750 \dagger$ \\
\hline \multirow[t]{2}{*}{ ASA score } & $<$ ASA3 & 119 & $96(80,7 \%)$ & $23(19,3 \%)$ & $0.089^{*}$ \\
\hline & $\geq A S A 3$ & 26 & $17(65,4 \%)$ & $9(34,6 \%)$ & \\
\hline \multirow[t]{2}{*}{ Comorbidities } & No & 101 & $84(83,2 \%)$ & $17(16,8 \%)$ & $0.021^{*}$ \\
\hline & Yes & 44 & $29(65,9 \%)$ & $15(34,1 \%)$ & \\
\hline \multirow[t]{2}{*}{ Blood transfusion } & No & 68 & $58(85,3 \%)$ & $10(14,7 \%)$ & $0.045^{*}$ \\
\hline & Yes & 77 & $55(71,4 \%)$ & $22(28,6 \%)$ & \\
\hline \multirow{2}{*}{$\begin{array}{l}\text { Splenectomyl } \\
\text { Splenopancreatectomy }\end{array}$} & No & 131 & $103(78,6 \%)$ & $28(21,2 \%)$ & \\
\hline & Yes & 14 & $10(71.4 \%)$ & $4(28.6 \%)$ & $0.537^{*}$ \\
\hline \multirow[t]{2}{*}{ LND } & D1 & 15 & $12(80 \%)$ & $3(20 \%)$ & $0.838^{*}$ \\
\hline & D1.5/D2 & 130 & $101(77.7 \%)$ & $29(22.3 \%)$ & \\
\hline \multirow[t]{2}{*}{ MVR } & No & 111 & $87(78,4 \%)$ & $24(21,6 \%)$ & $0.814^{*}$ \\
\hline & Yes & 34 & $26(76,5 \%)$ & $8(23,5 \%)$ & \\
\hline \multirow[t]{2}{*}{ Gastrectomy } & TG & 77 & $56(72,7 \%)$ & $21(27,3 \%)$ & $0.108^{*}$ \\
\hline & $P G$ & 68 & $57(83,8 \%)$ & $11(16,2 \%)$ & \\
\hline Operative time (mn) & & 145 & $189,50 \pm 51,50$ & $210 \pm 57,36$ & $0.055 \dagger$ \\
\hline \multirow{2}{*}{$\begin{array}{l}\text { Tumor size } \\
\text { (mean, } \mathrm{mm})\end{array}$} & $\geq 50 \mathrm{~mm}$ & 58 & $46(79.3 \%)$ & $12(20.7 \%)$ & $0.744^{*}$ \\
\hline & $<50 \mathrm{~mm}$ & 87 & $67(77 \%)$ & $20(23 \%)$ & \\
\hline \multirow[t]{2}{*}{ Depth of invasion } & $\mathrm{T} 4$ & 44 & $34(77.3 \%)$ & $10(22.7 \%)$ & $0.9^{*}$ \\
\hline & T1-T2-T3 & 101 & $79(78.2 \%)$ & $22(21.8 \%)$ & \\
\hline \multirow[t]{2}{*}{ Tumor location } & Distal & 77 & $62(80.5 \%)$ & $15(19.5 \%)$ & $0.424^{*}$ \\
\hline & Others & 68 & $51(75 \%)$ & $17(25 \%)$ & \\
\hline \multicolumn{6}{|c|}{$\begin{array}{l}\text { BMI: Body mass index, TG: total gastrectomy, PG : partial gastrectomy, MVR: multivisceral } \\
\text { resection, LND: lymph node dissection } \\
\dagger \text { test T of Student. } \\
\text { * test chi2 Pearson }\end{array}$} \\
\hline
\end{tabular}

Table 6: Univariate analysis of factors associated with the length of hospital stay

\begin{tabular}{|c|c|c|c|c|}
\hline \multicolumn{2}{|l|}{ Factors } & \multirow{2}{*}{$\begin{array}{l}\mathbf{N} \\
90\end{array}$} & \multirow{2}{*}{$\begin{array}{c}\begin{array}{c}\text { Length of hospital stay } \\
\text { (mean, days) * }\end{array} \\
14.41 \pm 5.538\end{array}$} & \multirow{2}{*}{$\begin{array}{c}\mathbf{P} \\
0.172\end{array}$} \\
\hline Age (years) & $\leq 65$ & & & \\
\hline & $>65$ & 55 & $16.16 \pm 9.845$ & \\
\hline \multirow[t]{2}{*}{ Gender } & Men & 93 & $14.49 \pm 7.933$ & 0.369 \\
\hline & Women & 52 & $14.33 \pm 6.618$ & \\
\hline \multirow[t]{2}{*}{ Comorbidities } & No & 101 & $13.81 \pm 5.096$ & 0.017 \\
\hline & Yes & 44 & $17.98 \pm 10.717$ & \\
\hline \multirow[t]{2}{*}{ Gastrectomy } & PG & 68 & $15.61 \pm 4.719$ & 0.381 \\
\hline & TG & 77 & $14.47 \pm 9.719$ & \\
\hline \multirow[t]{2}{*}{ MVR } & No & 111 & $14.95 \pm 8.157$ & 0.707 \\
\hline & Yes & 34 & $15.5 \pm 4.737$ & \\
\hline \multirow{2}{*}{$\begin{array}{l}\text { Splenectomyl } \\
\text { Splenopancreatectomy }\end{array}$} & No & 131 & $15.02 \pm 7.781$ & 0.145 \\
\hline & Yes & 14 & $15.57 \pm 3.857$ & \\
\hline \multirow[t]{2}{*}{ Lymphadenectomy } & D1/D1.5 & 51 & $14.52 \pm 8.32$ & 0.227 \\
\hline & D2 & 94 & $16.1 \pm 5.54$ & \\
\hline \multirow{2}{*}{$\begin{array}{l}\text { Postoperative } \\
\text { complications }\end{array}$} & No & 113 & $12.58 \pm 2.856$ & $<0.001$ \\
\hline & Yes & 32 & $23.91 \pm 11.292$ & \\
\hline \multirow[t]{6}{*}{ Grade Clavien Dindo } & No & 113 & $12.58 \pm 2.856$ & $<0.001$ \\
\hline & II & 21 & $20.43 \pm 4.664$ & \\
\hline & IIla & 3 & $26 \pm 8.544$ & \\
\hline & IIlb & 2 & $30 \pm 14.142$ & \\
\hline & Iva & 1 & 20 & \\
\hline & v & 5 & $35 \pm 23.082$ & \\
\hline \multirow[t]{2}{*}{ Fistula } & No & 135 & $14.11 \pm 5.186$ & 0.03 \\
\hline & Yes & 10 & $28.10 \pm 17.136$ & \\
\hline \multirow[t]{2}{*}{ Respiratory infection } & No & 132 & $14.52 \pm 7.457$ & 0.004 \\
\hline & Yes & 13 & $20.69 \pm 5.266$ & \\
\hline
\end{tabular}

TG: total gastrectomy, PG : partial gastrectomy, MVR: multivisceral resection

${ }^{*}$ test T of Student. 


\section{Discussion}

In our study, the rate of postoperative complications was $22.1 \%$ which seems consistent with the data in the literature.[1] However, we found a lower rate of major complications according to Clavien and Dindo classification $(7.6 \%)$, and this can be explained not only by the small number of the patients but also by a retrospective classification of complications based on data collected from medical records. The most common complications reported in the literature are respiratory complications occurring in $1.1 \%$ to $12.32 \%$ of cases[4], decompensation of chronic disease, and gastroparesis.

Anastomotic leakage occurring in 1 to $11.5 \%$, intraabdominal abscesses, pancreatic fistulas, intraperitoneal hemorrhages, postoperative occlusions, postoperative pancreatitis, and eviscerations were the most common surgical complications. [5, 6] However, Marrelli reported a higher rate of intra-abdominal infection of $14.2 \%$ with more extensive lymph node surgery.[7] In this study, we reported a rate of $9 \%$ of pneumonia, $6.9 \%$ of anastomotic fistula, and $4.2 \%$ of suppurative complications. According to Baiocchi, the postoperative mortality rate was also very variable. Western centers reported a mortality rate of $5 \%$, while Eastern centers reported a lower rate of $2 \%$.[1] In our series, postoperative deaths occurred in $3.4 \%$ of cases within an average of 30 days after the intervention, which seemed consistent with the literature data.

The extent of surgical resection, particularly the type of gastrectomy and lymphadenectomy, represent the most crucial factor of postoperative morbidity in gastric cancer.

Many studies evaluated the impact of extended lymphadenectomy on perioperative morbidity and mortality and the results were variable between the Asian series, particularly Japanese, and the Western series (Table 7). Two large randomized Western multicenter trials compared D1 and D2 lymphadenectomy: The British MRC ST01 study, conducted by Cuschieri[8, 9] and the Dutch trial conducted by Bonenkamp[10, 11] reported that D2 lymphadenectomy was associated with an increased rate of morbidity and included and a longer hospital stay. These two trials were included in a Cochrane meta-analysis[12] showing that D2 lymph node dissection had tripled the rate of mortality with a relative risk of 2.93 (95\% CI= 1,45-3.45) and concluded that the "excess mortality" of the D2 lymphadenectomy was related not only to spleno-pancreatectomy but also to the learning curve of the surgeons. However, several other randomized studies such as the German multicenter prospective study conducted by Siewert who had shown that D2 lymphadenectomy retrieving more than 25 lymph nodes was not significantly correlated with additional morbidity and mortality. [13] Although many studies reported a significant gain in survival with a D2-D3 lymphadenectomy, this extended lymph node dissection was associated to a higher rate of postoperative complications with a longer hospital stay, longer operative time and higher rate of blood transfusion.[14-16] However, the Japanese prospective study of Takeshi Sano et al. found that the incidence of serious complications was not different in the two groups.[17] Several reports have reported that splenectomy did not provide survival benefits and described a higher postoperative morbidity rate with and without splenectomy[18, 19], especially infectious complications [18] such as intra-abdominal abscess and pulmonary infections [20] and concluded that the use of prophylactic splenectomy to remove macroscopically negative lymph nodes near the splenic hilum in patients undergoing total gastrectomy for proximal gastric cancer should be avoided. However, in other randomized studies, D2 lymphadenectomy with splenectomy was not correlated to postoperative morbidity and mortality as well as the length of hospital stay and operative time.[17, 21, 22] In our series, no significant difference in duration of hospitalization or overall postoperative complication was found according to the extent of lymphadenectomy. Although splenectomy and left pancreatectomy exposed to a higher risk of complications $(28.6 \%$ vs $21.4 \%)$, the difference was not statistically significant $(p=0.537)$ with a comparable length of hospital stay. Our results can be explained by the low proportion of splenectomy and left pancreatectomy $(7.6 \%$ and $2.1 \%$ respectively) and by the selection of patients for extensive lymphadenectomy who were younger and with an ASA sore $<3$. However, in our study, extended lymphadenectomy led to an increased intraoperative morbidity with more blood transfusion and longer operative time.

Several studies investigated the impact of the type of gastrectomy on postoperative morbidity and mortality with variable results. In fact, since the extent of the gastrectomy depended on the site of the tumor and its size, most of the studies had essentially compared total gastrectomy to partial gastrectomy in distal tumors. These studies were included in a recent metaanalysis published in 2016 by Qi et al.[23], combining data from 5447 patients included in 10 retrospective studies and one prospective randomized study. In this meta-analysis, TG was associated with a higher risk of postoperative complications $(\mathrm{RR}=1.76,95 \% \mathrm{CI}=1.31$ $2.36, \mathrm{p}=0.0002)$ and especially more frequent intraabdominal abscesses $(\mathrm{RR}=3.41,95 \% \mathrm{CI}=1.21-9.63, \mathrm{p}$ $<0.05)$ compared to partial gastrectomy with a similar rate of postoperative mortality $(\mathrm{RR}=1.48,95 \% \mathrm{CI}=$ $0.90-2.44, p=0.12$ ). Moreover, the impact of the type gastrectomy on morbidity and mortality, regardless of the tumor location, has been reported in several other studies suggesting that TG is associated with higher rates of postoperative complications and morbidity, such as the Dutch trial $(\mathrm{OR}=2.04,95 \% \mathrm{CI}=1.01-3.79, \mathrm{p}=0.02)$. In the analysis of surgical morbidity and mortality of the Randomized "Critics" trial where 636 patients were included, total gastrectomy and oesophago-gastrectomy were independent risk factors for both surgical and medical postoperative complications $(\mathrm{OR}=1.88,95 \%$ $\mathrm{CI}=1.30-2.72, \mathrm{p}=0.001)$ with higher rates of surgical revision and longer hospital stay.[24] Nakagawa et al. performed a retrospective analysis of the risk factors of postoperative complications in 539 patients who were previously prospectively collected.[25]

In this study, total gastrectomy was the only independent factor of high-grade complications according to the Clavien and Dindo classification $(\mathrm{OR}=2.075,95 \% \mathrm{CI}$ $=0.26-0.896, p=0.021$. However, several other studies had reported comparable morbidity rates between the 
Table 7: Randomized trials comparing the impact of the extent of lymphadenectomy on surgical morbidity and mortality

\begin{tabular}{|c|c|c|c|c|c|}
\hline Study & LND & Morbidity & Mortality & $\begin{array}{l}\text { Hospital stay } \\
\text { (days) }\end{array}$ & $\begin{array}{c}\text { Blood } \\
\text { Transfusion }\end{array}$ \\
\hline $\begin{array}{l}\text { British MRC STO1 } \\
\text { Cuschieri et al }\end{array}$ & $\begin{array}{l}\text { D1: } N=200 \\
\text { D2: } N=200\end{array}$ & $\begin{array}{l}28 \% \\
46 \% \\
p<0,001\end{array}$ & $\begin{array}{l}6,5 \% \\
13 \% \\
p=0,015\end{array}$ & $\begin{array}{l}18 \text { (6 à } 101 \text { days) } \\
23 \text { (10 à } 147 \text { days) } \\
\mathbf{P = 0 , 0 1}\end{array}$ & $\mathrm{NE}$ \\
\hline $\begin{array}{l}\text { Dutch study } \\
\text { Bonenkamp et al }{ }^{10,11}\end{array}$ & $\begin{array}{l}\text { D1: } N=539 \\
\text { D2: } N=539\end{array}$ & $\begin{array}{l}25 \% \\
43 \% \\
p<0,001\end{array}$ & $\begin{array}{l}4 \% \\
10 \% \\
p<0,001\end{array}$ & $\begin{array}{l}18 \text { (7 à } 143 \text { days) } \\
25 \text { (7 à } 277 \text { days) } \\
\mathbf{p}<0,001\end{array}$ & $\mathrm{NE}$ \\
\hline $\begin{array}{l}\text { Scandinavian study } \\
\text { Danielson et al }{ }^{14}\end{array}$ & $\begin{array}{l}\text { D1: } N=114 \\
\text { D2: } N=109\end{array}$ & $\begin{array}{l}16,8 \% \\
33 \% \\
p=0,008\end{array}$ & $\begin{array}{l}1,8 \% \\
3,7 \% \\
p=0,438\end{array}$ & $\begin{array}{l}11 \text { (3 à } 66 \text { days) } \\
12 \text { ( } 6 \text { à } 69 \text { days) } \\
\mathbf{p = 0 , 0 1 2}\end{array}$ & $\begin{array}{l}400 \mathrm{ml} \\
550 \mathrm{ml} \\
p=0,047\end{array}$ \\
\hline $\begin{array}{l}\text { Taiwanese study } \\
\text { Wu et al }{ }^{15}\end{array}$ & $\begin{array}{l}\text { D1: } N=110 \\
\text { D3: } N=113\end{array}$ & $\begin{array}{l}4,5 \% \\
17,1 \% \\
\mathbf{p}<0,05\end{array}$ & $\mathrm{NE}$ & $\mathrm{NE}$ & $\mathrm{NE}$ \\
\hline $\begin{array}{l}\text { Chilean study } \\
\text { Butte } \mathrm{JM}^{16}\end{array}$ & $\begin{array}{l}\text { D1: } N=74 \\
\text { D3: } N=103\end{array}$ & $\begin{array}{l}4 \% \\
26 \% \\
p<0,05\end{array}$ & $\begin{array}{l}\% \\
5 \% \\
\mathbf{p}<0,05\end{array}$ & $\mathrm{NE}$ & NE \\
\hline $\begin{array}{l}\text { Japanese study } \\
\text { Takeshi Sano et al }{ }^{17}\end{array}$ & $\begin{array}{l}\text { D1: } N=263 \\
\text { D2: } N=260\end{array}$ & $\begin{array}{c}20,9 \% \\
28,1 \% \\
P=0,067\end{array}$ & $\begin{array}{c}0,8 \% \\
0,8 \% \\
\mathbf{p}=\mathbf{0 , 9 9}\end{array}$ & $\begin{array}{l}21 \text { days } \\
24 \text { days } \\
p<0,01\end{array}$ & $\begin{array}{c}14,1 \% \\
30 \% \\
\mathbf{p}<0,001\end{array}$ \\
\hline
\end{tabular}

\begin{tabular}{|c|c|c|c|c|c|}
\hline German study & D1: $N=558$ & $7,3 \%$ & $5 \%$ & $\mathrm{NE}$ & $\mathrm{NE}$ \\
\hline \multirow[t]{2}{*}{ Siewert ${ }^{13}$} & D2: $N=1096$ & $7,8 \%$ & $5,2 \%$ & & \\
\hline & & NS & NS & & \\
\hline Corean study & TG: $N=103$ & $8,7 \%$ & $1 \%$ & 11 (8 à 60 days) & $\mathrm{NE}$ \\
\hline \multirow[t]{2}{*}{ Yu et $a^{21}$} & TGS: $\mathrm{N}=104$ & $15,4 \% \%$ & $1,9 \%$ & 11 (1 à 71 days) & \\
\hline & & $p=0,142$ & $p=1$ & $p=0,272$ & \\
\hline Chilean study & TG: $N=97$ & $39 \%$ & $3,1 \%$ & 18,4 (8 à 81 days) & $\mathrm{NE}$ \\
\hline \multirow[t]{2}{*}{ Csendes et $\mathrm{al}^{20}$} & TGS: $\mathrm{N}=90$ & $50 \%$ & $4,4 \%$ & 21,6 (9 à 81 days) & \\
\hline & & $P=0,04$ & $p=0,7$ & $p=0,06$ & \\
\hline Italian study & D1: N=133 & $12 \%$ & $2,2 \%$ & 12,8 (8 à 78 days) & $\mathrm{NE}$ \\
\hline \multirow[t]{2}{*}{ Degiuli et $\mathrm{al}^{22}$} & D2: N=134 & $17,9 \%$ & $3 \%$ & 13,1 (7 à 79 days) & \\
\hline & & $P=0,178$ & $P=0,772$ & $p=0,732$ & \\
\hline
\end{tabular}

NE: Non evaluated, TG: total gastrectomy without splenectomy, GTS: total gastrectomy with splenectomy. LND: lymph node dissection

two surgical procedures. Indeed, the retrospective study of Park et al.[26] involving 719 patients did not report a significant difference between GT and GP regarding postoperative morbidity $(19.3 \%$ versus $13.6 \%$ respectively, $p=0.103$ ). However, Persiani found that the length of hospital stay exceeding ten days was particularly observed with total gastrectomy.[27] These results support the data from our series, where postoperative morbidity was not significantly correlated with the type of gastrectomy with a comparable rate of fistula and length of hospital stay even though the incidence of complications appeared to be higher in case of GT $(27.3 \%)$ compared to GP (16.2\%). However, TG was associated with higher perioperative morbidity with and increased rate of blood transfusion $(63.6 \%$ vs $41.2 \%, p=0.007)$ which is consistent with the results of the meta-analysis of Sun et al.[28] and also a longer operative time $(210 \mathrm{mn}$ vs $189.5 \mathrm{mn}, \mathrm{p}=0.015)$ which is consistent with the results of Gockel et al. and Papenfuss et al. $[29,30]$

The benefit of multivisceral resection (MVR) for locally advanced gastric ADK is controversial because of the increased mortality and morbidity. In the systematic review carried out by Brar[31] including 17 studies with 1343 patients, the morbidity rate varied between $11.8 \%$ and $90 \%$ and perioperative mortality ranged from 0 to $15 \%$. In contrast, in our study, MVR did not significantly increase the rate of postoperative complications (23.5\% vs $21.6 \%, \mathrm{p}=0.814)$ and fistula $(11.8 \%$ vs. $5.4 \%, \mathrm{p}=0.245)$ but was correlated to higher intraoperative morbidity with a significant lengthening of the operative time and an increase of blood transfusion requirement. Our findings were similar to that of the large multicenter and observational study published by Pacelli in 2013, who found that MVR was not associated with an increase in mortality $(\mathrm{p}=0.55)$ or morbidity $(33.9 \%$ vs $31.6 \%, \mathrm{p}=$ 0.38).[32]

The impact of intraoperative blood transfusions on 
short-term and long-term outcomes has been widely debated. In our study, blood transfusion had led to a significant increase in the rate of complication and fistula. Indeed, many authors had identified blood transfusions as an independent risk factor of major complications[30, 33] and supported the hypothesis that allogeneic blood transfusions induced immunosuppression that might increase the risk of infectious complications and leading to an extension of hospital stay.[34] Otherwise, in this study, transfused patients had a longer mean operation time than non-transfused patients (203.4 minutes vs183.82mn respectively, $p=0.054$ ), and the same results were also reported by Xiao et al.[34] and Ojima et al.[35] We also found that postoperative complication was correlated to the duration of surgical procedure and we stated that patients who developed an anastomotic fistula had a significantly longer operative time (238 min vs $190.77 \mathrm{~min}$, $p=0.006$ ) which is consistent with the results of the study of Migita et al.[5] who reported a significant correlation between anastomotic leakage and the duration of the surgical procedure (330 $\mathrm{min}$ vs $290 \mathrm{~min}, \mathrm{p}=0.0416)$ and the finding of Nakagawa et al. who reported that operating time exceeding $240 \mathrm{mn}$ was a risk factor for high-grade complications.[25]

Several intrinsic factors of postoperative morbidity of gastric cancer have been reported in the literature such as age, sex, nutritional status, and co-morbidities as well as tumor characteristics. The impact of gender and hormonal status on postoperative morbidity and mortality remains controversial. Although our results as well as those of Persiani et al.[27], Nakagawa et al.[25] and Lee et al.[36] did not support the correlation between gender and postoperative morbidity and mortality, the results of Critics trial[24] as well the 15-year results of the Dutch trial[37] have shown that men were exposed to a higher risk of post-operative complications and death. On the other hand, Sah et al. stated that women aged between 46 to 56 years were significantly predisposed to a higher risk of postoperative complications suggesting that hormonal instability related to menopausal status could result in a change of the host's response to stress and surgical trauma.[38]

Malnutrition can lead to the abnormal function of macrophages, neutrophils, and lymphocytes, which can inhibit the immune response and increase the surgical morbidity[39] and the incidence of major postoperative complications according to Clavien Dindoclassification.[36] In the recent study by Zheng et al. including 1976 patients, the group of malnourished patients with hypoproteinemia (412 patients) had a significantly higher complication rate $(21.4 \%$ vs $15.5 \%, \mathrm{p}=0.005)$.[40] Although we only recorded the level of proteinemia, we found that patients with hypoproteinemia had a higher rate of complications $(28.8 \%$ vs $18.3 \%, \mathrm{p}=0.066)$ and fistula $(11.5 \%$ vs $4.3 \%, \mathrm{p}=$ $0.168)$. Obesity (BMI> 25) is frequently associated with other co-morbidities such as diabetes, cardiovascular and respiratory diseases and according to The Japanese study by Ojima $\mathrm{T}$, overweight patients had a longer duration of intervention and a higher rate of postoperative complications (anastomotic release, pancreatic fistula). [41] However, in our series, postoperative complications were not significantly increased in patients with a BMI greater than $20 \mathrm{~kg} / \mathrm{m} 2$. These findings are comparable to those of several other studies which had not objectified a correlation between the weight of the patients and the specific surgical complications, and which had identified hypoalbuminemia as the only independent factor of morbidity.[25, 42]

Although in the studies by Persiani et al., Gil-Rendo et al., and Nakagawa et al., morbidity and mortality were correlated neither to age nor to co-morbidities and ASA score, but to the extensive surgery[25, 27, 43], other studies including that of Papenfuss reported that the implication of age as a factor of morbidity and mortality would most probably be related to the increased incidence of comorbidities with higher ASA score after 60 years and the alteration of the immune mechanisms.[30] In our series, the age of patients was not identified as a risk factor for morbidity even though we found that the average age of patients who died postoperatively was 75 years (range, 65-84 years). However, we found that postoperative complications were more frequent in the case of comorbidities with a higher rate of anastomotic fistula in diabetic patients and a longer duration of hospitalization. The predictive morbidity value of the ASA score in our study was similar to that of Lee et al. who reported a linear increase of surgical morbidity with ASA score without significant difference $(19.5 \%$ for ASA1, 24\% for the ASA2, and $31.4 \%$ if the ASA score was greater than or equal to 3; $\mathrm{p}=0.088) \cdot[36]$

Some authors had identified clinical and histological features of gastric tumors as intrinsic risk factors for postoperative morbidity. It has been reported that morbidity and mortality were higher in the upper and middle third gastric tumors.[43] On the contrary, in our study, there was no difference regarding postoperative morbidity according to the tumor location $(p=0.424)$. However, intraoperative morbidity was found to be higher in the proximal, middle, and total gastric tumors compared to distal tumors and the tumor site represents an independent risk factor of intraoperative blood transfusion. Also, the operative time was significantly shorter in distal tumors compared to other locations. These findings were like those of $\mathrm{Yu}$ et al. and Liu et al. who suggested that the incrimination of the proximal tumor site in postoperative morbidity would be more related to larger resections and more difficult anastomosis with more blood transfusion and longer operative time and not to the tumor location itself.[44, 45]

The correlation between the risk of postoperative complications and the tumor size can be explained by the fact that large tumors are associated with increased surgical technical difficulties, an extension of the type of gastrectomy, and the need for multi-visceral resections. $[33,42]$ Contrariwise, in our study, we found no significant difference in terms of post complications according to the tumor size. However, when analyzing the specific surgical complications, we found that tumors larger than $50 \mathrm{~mm}$ were significantly associated to a higher rate of fistula and an increase of blood transfusion requirement which is supported by the finding of Wang et al. who divided 513 patients into four groups according to the tumor size $(\leq 2, \leq 3, \leq 5,>5 \mathrm{~cm})$ and stated that the rate of postoperative complications was comparable between the four groups $(p=0.682)$ with a significant increase in blood transfusion with larger tumor (30\% for sizes $\leq 2,33.7 \%$ 
for sizes $\leq 3,46.3 \%$ for sizes $\leq 5$ and $63.9 \%$ for sizes $>5$ $\mathrm{cm}, \mathrm{p}<0.001)$.[46] The majority of authors as well as our finding had not demonstrated a strong correlation between postoperative morbidity and tumor stage and the depth of parietal invasion even if locally advanced tumors would be particularly associated with larger resections and more frequent surgical difficulties.[23, 24, 26, 27] However, we stated that surgery of T3-T4 tumors was associated with an increase of the intraoperative morbidity which was assessed by a longer operative time and more frequent blood transfusion supporting the results of Ojima et al. and Zhou et al.[35, 47]

Our study has some limitations not only its retrospective nature and the small number of included patients but also a lack of evaluation of the predictive factors of surgical mortality because of the low number of postoperative deaths.

\section{Conclusion}

Our study was able to analyze the intraoperative morbidity by determining the factors influencing the operative time and the intraoperative transfusions and suggested that it depends not only on the extent of the surgery and particularly the multi-visceral resections and the extension of the lymphadenectomy but also on the tumoral characteristics. In addition, the use of the Clavien and Dindo classification allowed an objective assessment of postoperative morbidity that depends on the patient's terrain and the association of comorbidities and tumor characteristics. We also identified the predictive factors of an anastomotic fistula which represented the most severe complication. Moreover, the occurrence of complications and their grade was the determinant of the length of postoperative hospital stay. Further studies focusing on late complications, functional results, and quality of life are needed to improve the surgical outcomes.

\section{Acknowledgements}

All authors thank Professor Ibtissem Abderrazek for the revision of the English language.

\section{Contributors}

HM, IZ, MAA, IBS, TBD, NM, LA, KR conceptualized and designed the study, acquired, and analyzed data, interpreted the study results, drafted the manuscript, and critically revised the final version of the manuscript.

\section{Funding}

This research did not receive any specific grant from funding agencies in the public, commercial, or not-forprofit sectors.

\section{Competing interests}

No benefits in any form have been received or will be received from a commercial party related directly or indirectly to the subject of this article.

\section{Availability of data and materials}

Further information is available from the corresponding author on reasonable request.

\section{Ethics approval}

The ethics review board approved this study and did not require informed consent from study participants since this was a strictly registry-based study.

\section{Provenance and peer review}

Not commissioned; externally peer reviewed.

\section{Open access}

This is an Open Access article distributed in accordance with the Creative Commons Attribution NonCommercial (CC BY-NC 4.0) license, which permits others to distribute, remix, adapt, build upon this work noncommercially, and license their derivative works on different terms, provided

the original work is properly cited and the use is non-commercial. See: http://creativecommons.org/ licenses/by-nc/4.0/

\section{References}

[1] Baiocchi GL, Giacopuzzi S, Marrelli D. Cutoff values of major surgical complications rates after gastrectomy. Updates in surgery. 2018;70:251-5

[2] Dindo D, Demartines N, Clavien P-A. Classification of surgical complications: a new proposal with evaluation in a cohort of 6336 patients and results of a survey. Annals of surgery. 2004;240:205.

[3] Association JGC. Japanese gastric cancer treatment guidelines 2010 (ver. 3). Gastric cancer. 2011;14:113-23.

[4] Tu R-H, Lin J-X, Li P, Xie J-W, Wang J-B, Lu J, et al. Prognostic significance of postoperative pneumonia after curative resection for patients with gastric cancer. Cancer Med. 2017;6:2757-65.

[5] Migita K, Takayama T, Matsumoto S, Wakatsuki K, Enomoto K, Tanaka T, et al. Risk factors for esophagojejunal anastomotic leakage after elective gastrectomy for gastric cancer. Journal of Gastrointestinal Surgery. 2012;16:1659-65.

[6] Xiao H, Xiao Y, Quan H, Liu W, Pan S, Ouyang Y. Intra-abdominal infection after radical gastrectomy for gastric cancer: incidence, pathogens, risk factors and outcomes. International Journal of Surgery. 2017:48:195-200.

[7] Marrelli D, Pedrazzani C, Neri A, Corso G, De Stefano A, Pinto E, et al. Complications after extended (D2) and superextended (D3) lymphadenectomy for gastric cancer: analysis of potential risk factors. Annals of surgical oncology. 2007;14:25-33.

[8] Cuschieri A, Joypaul V, Fayers P, Cook P, Fielding J, Craven J, et al. Postoperative morbidity and mortality after D1 and D2 resections for gastric cancer: preliminary results of the MRC randomised controlled surgical trial. The Lancet. 1996;347:995-9.

[9] Cuschieri A, Weeden S, Fielding J, Bancewicz J, Craven J, Joypaul $\mathrm{V}$, et al. Patient survival after D 1 and D 2 resections for gastric cancer: long-term results of the MRC randomized surgical trial. British journal of cancer. 1999;79:1522.

[10] Bonenkamp J, Songun I, Welvaart K, van de Velde C, Hermans J, Sasako M, et al. Randomised comparison of morbidity after D1 and D2 dissection for gastric cancer in 996 Dutch patients. The Lancet. 1995;345:745-8.

[11] Bonenkamp J, Hermans J, Sasako M, Welvaart K, Songun I, Meyer $S$, et al. Extended lymph-node dissection for gastric cancer. New England Journal of Medicine. 1999;340:908-14.

[12] McCulloch P, Nita ME, Kazi H, Gama-Rodrigues J. Extended versus limited lymph nodes dissection technique for adenocarcinoma of the stomach. Cochrane Database of Systematic Reviews. 2003.

[13] Siewert JR, Böttcher K, Stein HJ, Roder JD. Relevant prognostic factors in gastric cancer: ten-year results of the German Gastric Cancer Study. Annals of surgery. 1998;228:449.

[14] Danielson H, Kokkola A, Kiviluoto T, Sirén J, Louhimo J, Kivilaakso E, et al. Clinical outcome after D1 vs D2-3 gastrectomy for treatment of gastric cancer. Scandinavian Journal of Surgery. 2007;96:35-40.

[15] Wu C-W, Chang I-S, Lo S-s, Hsieh M-C, Chen J-H, Lui W-Y, et al. Complications following D3 gastrectomy: post hoc analysis of a randomized trial. World journal of surgery. 2006;30:12-6.

[16] Butte JM, Kerrigan N, Waugh E, Meneses M, Parad H, Visscher A, et al. Complications and mortality of extended gastrectomy for gastric cancer. Revista medica de Chile. 2010;138:1487-94.

[17] Sano T, Sasako M, Yamamoto S, Nashimoto A, Kurita A, Hiratsuka $M$, et al. Gastric cancer surgery: morbidity and mortality results from a prospective randomized controlled trial comparing D2 and extended para-aortic lymphadenectomy-Japan Clinical Oncology Group study 
9501. Journal of clinical oncology. 2004;22:2767-73.

[18] Brady MS, Rogatko A, Dent LL, Shiu MH. Effect of splenectomy on morbidity and survival following curative gastrectomy for carcinoma. Archives of surgery. 1991;126:359-64.

[19] Kwon SJ. Prognostic impact of splenectomy on gastric cancer: results of the Korean Gastric Cancer Study Group. World journal of surgery. 1997;21:837-44

[20] Csendes A, Burdiles P, Rojas J, Braghetto I, Diaz JC, Maluenda F. A prospective randomized study comparing D2 total gastrectomy versus D2 total gastrectomy plus splenectomy in 187 patients with gastric carcinoma. Surgery. 2002;131:401-7.

[21] Yu W, Choi G, Chung H. Randomized clinical trial of splenectomy versus splenic preservation in patients with proximal gastric cancer. British Journal of Surgery: Incorporating European Journal of Surgery and Swiss Surgery. 2006;93:559-63.

[22] Degiuli M, Sasako M, Ponti A. Morbidity and mortality in the Italian Gastric Cancer Study Group randomized clinical trial of D1 versus D2 resection for gastric cancer. British Journal of Surgery. 2010;97:643-9.

[23] Qi J, Zhang P, Wang Y, Chen H, Li Y. Does total gastrectomy provide better outcomes than distal subtotal gastrectomy for dista gastric cancer? A systematic review and meta-analysis. PloS one. 2016;11:e0165179.

[24] Claassen Y, Hartgrink H, Dikken J, de Steur W, van Sandick J, van Grieken N, et al. Surgical morbidity and mortality after neoadjuvant chemotherapy in the CRITICS gastric cancer trial. European Journal of Surgical Oncology. 2018;44:613-9.

[25] Nakagawa M, Kojima K, Inokuchi M, Kato K, Sugita H, Otsuki S, et al. Identification of frequency, severity and risk factors of complications after open gastrectomy: Retrospective analysis of prospectively collected database using the Clavien-Dindo classification. Journal of medical and dental sciences. 2016;63:53-9.

[26] Park DJ, Lee HJ, Kim HH, Yang HK, Lee KU, Choe KJ. Predictors of operative morbidity and mortality in gastric cancer surgery. The British journal of surgery. 2005:92:1099-102.

[27] Persiani R, Antonacci V, Biondi A, Rausei S, La Greca A, Zoccali M, et al. Determinants of surgical morbidity in gastric cancer treatment. Journal of the American College of Surgeons. 2008;207:13-9.

[28] Sun C, Wang Y, Yao HS, Hu ZQ. Allogeneic blood transfusion and the prognosis of gastric cancer patients: systematic review and meta-analysis. International journal of surgery (London, England). 2015;13:102-10.

[29] Gockel I, Pietzka S, Gönner U, Hommel G, Junginger T. Subtotal or total gastrectomy for gastric cancer: impact of the surgical procedure on morbidity and prognosis-analysis of a 10-year experience. Langenbeck's archives of surgery. 2005;390:148-55.

[30] Papenfuss WA, Kukar M, Oxenberg J, Attwood K, Nurkin S, Malhotra U, et al. Morbidity and mortality associated with gastrectomy for gastric cancer. Annals of surgical oncology. 2014;21:3008-14.

[31] Brar SS, Seevaratnam R, Cardoso R, Yohanathan L, Law C, Helyer $\mathrm{L}$, et al. Multivisceral resection for gastric cancer: a systematic review. Gastric Cancer. 2012;15:100-7.

[32] Pacelli F, Cusumano G, Rosa F, Marrelli D, Dicosmo M, Cipollari $\mathrm{C}$, et al. Multivisceral resection for locally advanced gastric cancer: an Italian multicenter observational study. JAMA surgery. 2013;148:35360.

[33] Tran TB, Worhunsky DJ, Norton JA, Squires MH, Jin LX, Spolverato G, et al. Multivisceral resection for gastric cancer: results from the US gastric cancer collaborative. Annals of surgical oncology. 2015;22:840-7.

[34] Xiao H, Quan H, Pan S, Yin B, Luo W, Huang G, et al. Impact of peri-operative blood transfusion on post-operative infections after radical gastrectomy for gastric cancer: a propensity score matching analysis focusing on the timing, amount of transfusion and role of leukocyte depletion. Journal of cancer research and clinical oncology. 2018;144:1143-54.

[35] Ojima T, Iwahashi M, Nakamori M, Nakamura M, Naka T, Katsuda $\mathrm{M}$, et al. Association of allogeneic blood transfusions and long-term survival of patients with gastric cancer after curative gastrectomy. Journal of Gastrointestinal Surgery. 2009;13:1821.

[36] Lee K-G, Lee H-J, Yang J-Y, Oh S-Y, Bard S, Suh Y-S, et al. Risk factors associated with complication following gastrectomy for gastric cancer: retrospective analysis of prospectively collected data based on the Clavien-Dindo system. Journal of Gastrointestinal Surgery. 2014;18:1269-77.

[37] Songun I, Putter H, Kranenbarg EM-K, Sasako M, van de Velde CJ. Surgical treatment of gastric cancer: 15-year follow-up results of the randomised nationwide Dutch D1D2 trial. The lancet oncology. 2010;11:439-49.

[38] Sah B, Zhu Z, Wang X, Yang Q, Chen M, Xiang M, et al. Postoperative complications of gastric cancer surgery: female gender at high risk. European journal of Cancer care. 2009;18:202-8.

[39] Cunningham-Rundles S, McNeeley DF, Moon A. Mechanisms of nutrient modulation of the immune response. Journal of Allergy and
Clinical Immunology. 2005;115:1119-28.

[40] Zheng H-L, Lu J, Li P, Xie J-W, Wang J-b, Lin J-X, et al. Effects of preoperative malnutrition on short-and long-term outcomes of patients with gastric cancer: can we do better? Annals of surgical oncology. 2017;24:3376-85.

[41] Ojima T, Iwahashi M, Nakamori M, Nakamura M, Naka T, Ishida $\mathrm{K}$, et al. Influence of overweight on patients with gastric cancer after undergoing curative gastrectomy: an analysis of 689 consecutive cases managed by a single center. Archives of Surgery. 2009;144:351-8.

[42] Kang SC, Kim HI, Kim MG. Low Serum Albumin Level, Male Sex, and Total Gastrectomy Are Risk Factors of Severe Postoperative Complications in Elderly Gastric Cancer Patients. J Gastric Cancer. 2016;16:43-50.

[43] Gil-Rendo A, Hernandez-Lizoain JL, Martinez-Regueira F, Sierra Martinez A, Rotellar Sastre F, Cervera Delgado M, et al. Risk factors related to operative morbidity in patients undergoing gastrectomy for gastric cancer. Clinical \& translational oncology : official publication of the Federation of Spanish Oncology Societies and of the National Cancer Institute of Mexico. 2006;8:354-61.

[44] Yu X, Hu F, Li C, Yao Q, Zhang H, Xue Y. Clinicopathologic characteristics and prognosis of proximal and distal gastric cancer. OncoTargets and therapy. 2018;11:1037-44.

[45] Liu K, Zhang W, Chen X, Chen X, Yang K, Zhang B, et al. Comparison on Clinicopathological Features and Prognosis Between Esophagogastric Junctional Adenocarcinoma (Siewert II/III Types) and Distal Gastric Adenocarcinoma: Retrospective Cohort Study, a Single Institution, High Volume Experience in China. Medicine. 2015;94:e1386.

[46] Wang X, Wan F, Pan J, Yu GZ, Chen Y, Wang JJ. Tumor size: a nonneglectable independent prognostic factor for gastric cancer. Journal of surgical oncology. 2008;97:236-40.

[47] Zhou HY, Yi W, Wang J, Zhang J, Wang WJ, Hu ZQ. Association of perioperative allogeneic blood transfusions and prognosis of patients with gastric cancer after curative gastrectomy. American journal of surgery. 2014;208:80-7. 